\title{
Rolapitant Is a Reversible Inhibitor of CYP2D6 ${ }^{[\mathrm{s}}$
}

\author{
Sarah M. Glass, Sabrina M. Leddy, Michael C. Orwin, Garret P. Miller, Kyle A. Furge, \\ and Laura Lowe Furge
}

Department of Chemistry, Kalamazoo College, Kalamazoo, Michigan

Received December 17, 2018; accepted March 25, 2019

\begin{abstract}
Rolapitant [(Varubi), 5S,8S)-8-[[(1R)-1-[3,5 bis(trifluoromethyl phenyl]ethoxy]methyl]-8-phenyl-1,7-diazaspiro[4.5]decan-2-one] is a high-affinity NK1 receptor antagonist that was approved in September $\mathbf{2 0 1 5}$ as a treatment for nausea and vomiting caused by chemotherapy. In vivo rolapitant moderately inhibits CYP2D6 for at least 7 days after one $\mathbf{1 8 0} \mathbf{~ m g}$ dose. Due to the long inhibition time, we investigated rolapitant as a possible mechanism-based inactivator of CYP2D6. Rolapitant docked in the active site of CYP2D6 and displayed type I binding to CYP2D6 with a $K_{\mathrm{s}}$ value of $1.2 \pm 0.4 \mu \mathrm{M}$. However, in NADPH-, time-, and concentration-dependent assays of CYP2D6 activity, no evidence for mechanism-based inactivation and no metabolites of rolapitant were observed. Stopped-flow binding studies yielded a $k_{\text {on }} / k_{\text {off }}\left(K_{\mathrm{d}}\right)$ value of $6.2 \mu \mathrm{M}$. The $\mathrm{IC}_{50}$ value for rolapitant inhibition of CYP2D 6 activity was $24 \mu \mathrm{M}$, suggesting that
\end{abstract}

\section{Introduction}

Rolapitant [5S,8S)-8-[[(1R)-1-[3,5 bis(trifluoromethyl phenyl] ethoxy]methyl]-8-phenyl-1,7-diazaspiro[4.5]decan-2-one] is a highaffinity NK1 receptor antagonist recently approved under the name Varubi as a treatment for nausea and vomiting caused by chemotherapy by the U.S. Food and Drug Administration (FDA) (Fig. 1) (Food and Drug Administration, 2015; Varubi, 2015). Rolapitant is metabolized in vivo primarily by CYP3A4 to form the major metabolite M19 (C4-pyrrolidinehydroxylated rolapitant) (Food and Drug Administration, 2015). However, in animal studies the majority of the drug is excreted unmetabolized ( $\sim 14 \%$ in urine and $\sim 73 \%$ in feces over 6 weeks) (Varubi, 2015).

One advantage of rolapitant over other available antiemetics is that it does not inhibit or induce CYP3A4 (Poma et al., 2013; Food and Drug Administration, 2015), which is the cytochrome P450 (P450) most involved in the metabolism of pharmaceutical drugs (Guengerich, 2015). Given the lack of CYP3A4 inhibition, rolapitant is believed to reduce drug-drug interactions (DDIs) (Olver, 2015). However, rolapitant

This work was supported by the National Institutes of Health [Grant 1R15GM086767-02] (to L.L.F.); a grant to Kalamazoo College from the Howard Hughes Medical Institute [Grant 52006304] through the Precollege and Undergraduate Science Education Program; and the Cook and Varney Funds of Kalamazoo College.

This work was presented in part at the 2017 American Society for Biochemistry and Molecular Biology Annual Meeting, Chicago, IL, and the 2018 Great Lakes Drug Metabolism and Disposition Discussion Group Annual Meeting, Indianapolis, IN.

https://doi.org/10.1124/dmd.118.085928.

S This article has supplemental material available at dmd.aspetjournals.org. inhibition is not due to tight binding of rolapitant to CYP2D6. By Lineweaver-Burk analysis, rolapitant behaved as a mixed, reversible inhibitor. The $K_{\mathrm{i}}$ values of 20 and $34 \mu \mathrm{M}$ were determined by Dixon analysis, with bufuralol and dextromethorphan as reporter substrates, respectively, and drug-drug interaction modeling did not predict the reported in vivo inhibition. The interaction of rolapitant with CYP2D6 was also examined in 1 microsecond molecular dynamics simulations. Rolapitant adopted multiple low-energy binding conformations near the active site, but at distances not consistent with metabolism. Given these findings, we do not see evidence that rolapitant is a mechanismbased inactivator. Moreover, the reversible inhibition of CYP2D6 by rolapitant may not fully account for the moderate inhibition described in vivo.

has been shown to moderately inhibit the activity of CYP2D6 for at least 7 days after only one $180 \mathrm{mg}$ dose (Food and Drug Administration, 2015; Wang et al., 2019).

Due to the long inhibition time and increased area under the curve ( 3-fold higher with dextromethorphan as victim drug) (Varubi, 2015) in clinical studies, rolapitant was investigated as a possible substrate and mechanism-based inactivator of CYP2D6. Rolapitant has basic nitrogen and aromatic rings with a molecular weight of $500.5 \mathrm{~g} / \mathrm{mol}$ (Fig. 1). Of the over 90 substrates in the CYP2D6 small molecule kinetics database, only two substrates for CYP2D6 [e.g., amiodarone $(645.3 \mathrm{~g} / \mathrm{mol})$ and ritonavir $(720.9 \mathrm{~g} / \mathrm{mol})]$ have molecular weights larger than that of rolapitant (Chico et al., 2009).

Our initial studies showed that rolapitant could dock in the active site of CYP2D6 in an orientation consistent with metabolism. Furthermore, spectral binding titrations produced type 1 binding, consistent with binding of a substrate (vide infra). The goal of the present study was to examine the mechanism by which rolapitant achieves long-term inhibition of CYP2D6 in vivo.

\section{Materials and Methods}

Chemicals. Rolapitant was purchased from AdooQ Bioscience (Irvine, CA) and reconstituted in DMSO for use in the assays described subsequently. Ultrapure solvents [water, acetonitrile (ACN), and methanol] for mass spectrometry were purchased from EMD Chemicals, Inc. (Gibbstown, NJ). All other solvents were high-performance liquid chromatography-grade and purchased from Sigma-Aldrich (St. Louis, MO). Bufuralol (mixture of enantiomers) and hydroxyl-bufuralol were purchased from Toronto Research Chemical (North York, ON, Canada). Potassium phosphate, NADPH, dextromethorphan, dextrorphan, and all other reagents were purchased from Sigma-Aldrich.

ABBREVIATIONS: ACN, acetonitrile; DDI, drug-drug interaction; FDA, Food and Drug Administration; P450, cytochrome P450; rolapitant, 5S,8S)8-[[(1R)-1-[3,5 bis(trifluoromethyl phenyl]ethoxy]methyl]-8-phenyl-1,7-diazaspiro[4.5]decan-2-one; $t_{\mathrm{R}}$, residence time. 


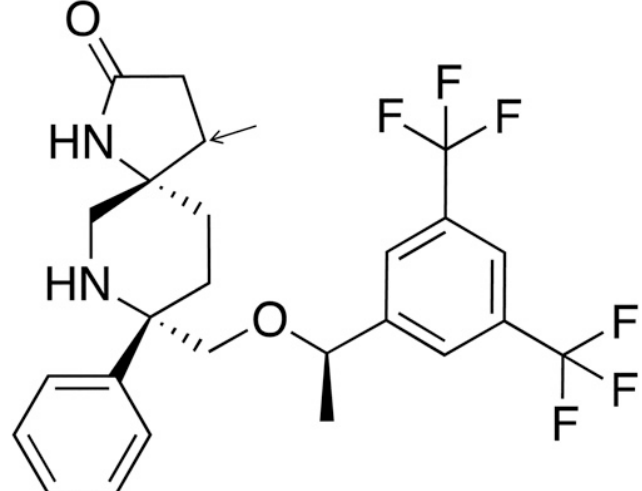

Fig. 1. Structure of rolapitant. Rolapitant contains a spirolactam ring structure with three chiral centers and has a molecular weight of $500.2 \mathrm{~g} / \mathrm{mol}$. The arrow indicates the site of hydroxylation by CYP3A4 to form the major metabolite, M19.

Enzymes. Supersomes (Corning Life Sciences, Tewksbury, MA) were used in all assays except spectral binding titrations and stopped-flow spectroscopy. For spectral binding titrations and stopped-flow spectroscopy, purified CYP2D6 and CYP3A4 were used; expression and purification were as described elsewhere (Gillam et al., 1993, 1995; Hanna et al., 2001; Glass et al., 2018).

Time-Dependent Inactivation of CYP2D6 Supersomes with Rolapitant. Four primary reaction, mixtures of CYP2D6 supersomes $(20 \mathrm{pmol})$ in potassium phosphate buffer $(\mathrm{pH} 7.4,100 \mathrm{mM})$ were incubated in a $37^{\circ} \mathrm{C}$ shaking bath for 3 minutes. Two of these mixtures contained rolapitant $(5 \mu \mathrm{M})$ and two contained DMSO for solvent vehicle control. After 3 minutes, two primary reaction mixtures (one with and one without rolapitant) were initiated with NADPH $(1 \mathrm{mM})$. The other two received water as a control. The final reaction volume was $100 \mu \mathrm{l}$. Aliquots $(10 \mu \mathrm{l})$ of the primary reaction were removed at various time points $(0,1$, $5,10,15$, and 30 minutes) and transferred to a secondary reaction containing bufuralol $(100 \mu \mathrm{M})$, NADPH $(1 \mathrm{mM})$, and potassium phosphate buffer $(\mathrm{pH} 7.4$, $100 \mathrm{mM}$ ). The final secondary reaction volume was $200 \mu \mathrm{l}$. Reactions were quenched with $30 \mu \mathrm{l} \mathrm{ACN}$ after 10.5 minutes. Samples were then centrifuged $(16,100 \mathrm{~g})$ for 5 minutes, and $10 \mu \mathrm{l}$ of the supernatant was injected onto a Waters Symmetry C18 column ( $5 \mu \mathrm{m}, 3.9 \times 150 \mathrm{~mm}$; Waters Corporation, Milford, MA) connected to a Waters e2965 high-performance liquid chromatography system paired with a Waters 2475 fluorescence detector. The instrument method and mobile phase were the same as previously described with bufuralol (Nagy et al., 2011; Glass et al., 2018). The 1'-OH-bufuralol peak area was converted to concentration using a standard curve.

Concentration-Dependent Inactivation of CYP2D6 Supersomes with Rolapitant. Ten primary reaction mixtures of 2D6 supersomes (20 pmol), potassium phosphate buffer ( $\mathrm{pH} 7.4,100 \mathrm{mM})$, and varying concentrations of rolapitant $(0-40 \mu \mathrm{M})$ were incubated in a $37^{\circ} \mathrm{C}$ shaking bath for 3 minutes, as indicated in the figure legends. The $0 \mu \mathrm{M}$ rolapitant reaction contained DMSO as a control. After 3 minutes, five of the reactions (one for each concentration of rolapitant) were initiated with NADPH $(1 \mathrm{mM})$, while the other five received water as a control. The final reaction volume was $50 \mu \mathrm{l}$. Aliquots $(10 \mu \mathrm{l})$ of the primary reaction were removed 20 minutes after initiation and transferred to secondary reaction mixtures containing NADPH $(1 \mathrm{mM})$, bufuralol $(100 \mu \mathrm{M})$, and potassium phosphate buffer $(\mathrm{pH} \mathrm{7.4,100} \mathrm{mM).} \mathrm{The} \mathrm{final} \mathrm{secondary} \mathrm{reaction}$ volume was $200 \mu \mathrm{l}$. Reactions were quenched with $30 \mu \mathrm{l}$ of cold ACN after 10 minutes. Samples were then centrifuged $(16,100 \mathrm{~g})$ for 5 minutes and $10 \mu \mathrm{l}$ of the supernatant was analyzed as described previously.

Rolapitant Metabolites Formed by $2 \mathrm{D6}$ and 3A4 Supersomes. Rolapitant $(50 \mu \mathrm{M})$ in potassium phosphate buffer $(\mathrm{pH} 7.4,100 \mathrm{mM})$ was combined with either CYP2D6 or CYP3A4 supersomes $(20 \mathrm{pmol})$. The mixtures were preincubated for 3 minutes at $30^{\circ} \mathrm{C}$ in a shaking bath before initiation by NADPH $(1 \mathrm{mM})$. The final reaction volume was $100 \mu \mathrm{l}$. The reactions proceeded for 30 minutes before being quenched with $20 \mu \mathrm{l} \mathrm{ACN}$ and placed on ice. The samples were centrifuged $(16,100 \mathrm{~g})$ for 5 minutes, and $10 \mu \mathrm{l}$ of the supernatant was injected onto a Kinetex C18 column $(2.6 \mu \mathrm{m}, 100 \AA, 100 \times 2.10 \mathrm{~mm}$; Phenomenex, Torrance, CA) connected to a Waters Alliance 2690 high-performance liquid chromatography system. A gradient separation system was achieved using
$0.1 \%$ formic acid in mass spectrometry-grade water (solvent $\mathrm{A}$ ) and acetonitrile (solvent B). The initial conditions were $90 \% \mathrm{~A}$ and $10 \% \mathrm{~B}$. These conditions were held for 5 minutes. At 30 minutes, the conditions were $10 \%$ A and $90 \% \mathrm{~B}$. These conditions were held for 5 minutes. At 40 minutes, the gradient returned to the initial conditions, which were held for an additional 10 minutes. The total run time was 50 minutes, with a flow rate of $0.1 \mathrm{ml} / \mathrm{min}$. The samples were analyzed using a Thermo-Fisher Scientific LXQ mass spectrometer in positive ion mode (Waltham, MA).

Binding Studies. Spectral binding titrations for determination of $K_{s}$ were performed as previously described (Nagy et al., 2011). Briefly, purified CYP2D6 $(1 \mu \mathrm{M})$ or CYP3A4 $(2 \mu \mathrm{M})$ in potassium phosphate buffer $(\mathrm{pH} 7.4,100 \mathrm{mM})$ was divided evenly between two microquartz cuvettes. A baseline was taken from 350 to $500 \mathrm{~nm}$ with a Cary 300 spectrophotometer (Agilent, Santa Clara, CA). Rolapitant (0.05-150 $\mu \mathrm{M}$, final) was then added to the sample cuvette while an equal volume of DMSO was added to the reference cuvette. Spectra from 350 to $500 \mathrm{~nm}$ were recorded after each addition. The total amount of DMSO added did not exceed $2 \%(\mathrm{v} / \mathrm{v})$. Titrations with both CYP2D6 and CYP3A4 were type I. Differences in absorbance between 390 and $420 \mathrm{~nm}$ were plotted against concentrations of ligand and fit with the quadratic velocity equation, or tight-binding equation: $[C Y P \cdot$ rolapitant $]=0.5\left(K_{\mathrm{s}}+\mathrm{E}_{\mathrm{t}}+\mathrm{S}_{\mathrm{t}}\right)-\left[0.25\left(K_{\mathrm{s}}+\mathrm{E}_{\mathrm{t}}+\mathrm{S}_{\mathrm{t}}\right)^{2}-\mathrm{E}_{\mathrm{t}} \mathrm{S}_{\mathrm{t}}\right]^{1 / 2}$, where $\mathrm{S}$ represents the substrate concentration, $\mathrm{E}$ is the total enzyme concentration, and $K_{\mathrm{s}}$ is the spectral dissociation constant for the reaction CYP + rolapitant $\rightarrow$ CYP - rolapitant. The dissociation constant, $K_{\mathrm{s}}$, was determined using KaleidaGraph software (Synergy Software, Reading, PA). Spectra for each concentration were adjusted such that each scan intersected at zero absorbance at the isobestic point $(407 \mathrm{~nm})$.

Stopped-flow spectroscopy was used to determine $k_{\text {on }}$ and $k_{\text {off }}$ using a rapid scanning monochrometer OLIS RSM-1000 stopped-flow instrument (On-Line Instrument Systems) in the laboratory of Dr. F. P. Guengerich (Vanderbilt University). Stopped-flow sample syringes contained $50 \mathrm{mM}$ potassium phosphate buffer ( $\mathrm{pH} 7.4)$ and either $4 \mu \mathrm{M}$ purified CYP2D6 or rolapitant $(2-100 \mu \mathrm{M})$ diluted in water. Equal volumes from both syringes were injected in the sample cell $(4 \times 20 \mathrm{~mm})$ at room temperature with a final volume of $2 \mathrm{ml}$. Absorbance spectra from 350 to $500 \mathrm{~nm}$ were recorded at 1-millisecond intervals for a total of 4 seconds. Data were not collected during the initial 4-millisecond mix time. Data subsets at the absorbance maxima and minima (390 and $420 \mathrm{~nm}$, respectively) were created on the OLIS software for each rolapitant concentration and then subtracted to create the composite absorbance changes over time $\left(\Delta A_{390-420}\right)$. At least three replicates of each ligand concentration were averaged and plotted over time. The experiment parameters [enzyme (E) and ligand (L)] were entered and the data were fit to a minimal kinetic model (single-step second-order reaction, $\mathrm{E}+\mathrm{L} \rightarrow \mathrm{EL}$ ) to estimate binding constants using KinTek Global Kinetic Explorer software (KinTek, Snow Shoe, PA). The $K_{\mathrm{d}}$ value for rolapitant dissociation from CYP2D6 was calculated from $k_{\text {off }} / k_{\text {on }}$. Rolapitant residence time $\left(t_{\mathrm{R}}\right)$ was calculated as $1 / k_{\text {off }}$.

Determination of $K_{\mathrm{i}}$ for Rolapitant with CYP2D6 Supersomes. Reaction mixtures contained rolapitant $(0-100 \mu \mathrm{M})$, bufuralol $(5-100 \mu \mathrm{M})$ or dextromethorphan (5-100 $\mu \mathrm{M})$, CYP2D6 supersomes ( 2 pmol), and potassium phosphate buffer ( $\mathrm{pH} 7.4,100 \mathrm{mM})$, as indicated in the figure legends. The reaction mixture was incubated in a $37^{\circ} \mathrm{C}$ shaking bath for 3 minutes and then initiated with NADPH $(1 \mathrm{mM})$. The final reaction volume was $100 \mu \mathrm{l}$. Reactions were quenched with $20 \mu \mathrm{ACN}$ and placed on ice after a time determined to be in the linear range for product formation. Samples were then centrifuged $(16,100 \mathrm{~g})$ for 5 minutes, and $10 \mu \mathrm{l}$ of the supernatant was analyzed as previously described (Glass et al., 2018). Lineweaver-Burk and Dixon plots were created using KaleidaGraph (Synergy Software). All experiments were run in quadruplicate.

Determination of $\mathbf{I C}_{\mathbf{5 0}}$. CYP2D6 supersomes $(2 \mathrm{pmol})$ were incubated with dextromethorphan $(10 \mu \mathrm{M})$ and quinidine $(0-50 \mu \mathrm{M})$ or rolapitant $(0-100 \mu \mathrm{M})$ in potassium phosphate buffer, $\mathrm{pH} 7.4(100 \mathrm{mM})$. Quinidine was dissolved in methanol and rolapitant in DMSO. Incubations contained no more than $2 \%$ organic (v/v). The final reaction volume was $100 \mu$ l. Reactions were incubated in a $37^{\circ} \mathrm{C}$ shaking bath for 3 minutes before being initiated with NADPH $(1 \mathrm{mM})$. Reactions were quenched with $20 \mu \mathrm{l}$ of cold ACN after 10 minutes. Samples were then centrifuged $(16,100 \mathrm{~g})$ for 5 minutes and dextrorphan product formation was measured as previously described (Glass et al., 2018).

Molecular Dynamics with Rolapitant. Simulation setup and initialization was performed largely as described previously (de Waal et al., 2014). Briefly, for 
system setup a 2.8 A crystal structure of CYP2D6 bound to prinomastat (PDB ID: $3 \mathrm{QM} 4$, chain A) was used as starting protein and heme coordinates with the exception that the prinomastat ligand was removed. The AMBER99SB and generalized AMBER force fields (GAFF) were used for protein and heme model parameters along with quantum mechanically derived parameters for the oxygen complex in a resting high-spin compound I state (Shahrokh et al., 2012). The protein + heme + oxygen system was solvated in a $10 \AA$ pad of TIP3P waters and neutralized. Restrained electrostatic potential charges for protonated rolapitant $(+1)$ were derived using the Gaussian09_E.01 option from R.E.D. Server (Vanquelef et al., 2011). AutoDock Vina (http://autodock.scripps.edu) (Morris et al., 1998; Huey et al., 2007; Trott and Olson, 2010) was used to identify an initial pose of protonated rolapitant $(+1)$ near the CYP2D6 heme to produce a combined protein + heme + oxygen + ligand system. The initial pose of rolapitant was chosen by visual inspection of the lowest energy poses.

Following system setup, the system was solvent energy minimized, full system energy minimized, heated, and NPT (constant number, pressure, and temperature) and NVT (constant number, volume, and temperature) pressurized as previously described (de Waal et al., 2014) using pmemd.cuda (Amber16). One-microsecond molecular dynamics simulations were performed using a version of pmemd.cuda that was modified to incorporate an adaptive biasing potential as described by Dickson et al. (2016). While the initial adaptive biasing potential implementation was integrated with GROMACS (Dickson et al., 2016), our version was modified for use with Amber16 and generously provided to us by P. de Waal (Van Andel
Research Institute, Grand Rapids, MI). In these types of simulations, a very small amount of energy is deposited at the space where the ligand currently occupies. Accumulation of small energy hills during the simulation encourages the ligand to move; therefore, more configuration space is sampled during a given simulation run. Without the use of biasing strategies (e.g., filling in low-energy wells), the likelihood of the ligand moving significantly within the active site or egressing during a 1-microsecond simulation is extremely small. In addition, tracking where energy is deposited can be used to estimate rolapitant's low-energy binding positions. A schematic of our approach to adaptive biasing simulations is shown in Supplemental Fig. 1. To prevent ligand diffusion, a spherical ligand restraint was included such that the sphere was at least $6 \AA$ from any CYP2D6 atom (Supplemental Fig. 1, A and B). Biasing parameters were set at $c=0.01$ and $b=0.8$. The simulation was run for $5 \times 10^{8}$ steps with each step representing 2 femtoseconds. Each simulation took approximately 10 days to complete on a NVIDIA GeForce GTX 980 Ti GPU.

Tracking the position of every rolapitant atom during the adaptive biasing simulation run is computationally complex. To reduce this computational complexity, adaptive biasing schemes track a courser grained representation of the ligand by collecting different parts of the ligand into a single variable. In our models, the root-mean-square deviations of two collections of atoms (protonated rolapitant collective variables 1 and 2) are tracked by the adaptive biasing algorithm versus a reference point near the heme (Supplemental Fig. 1, C-E). These collective variable atoms were picked such that the biasing potential did not
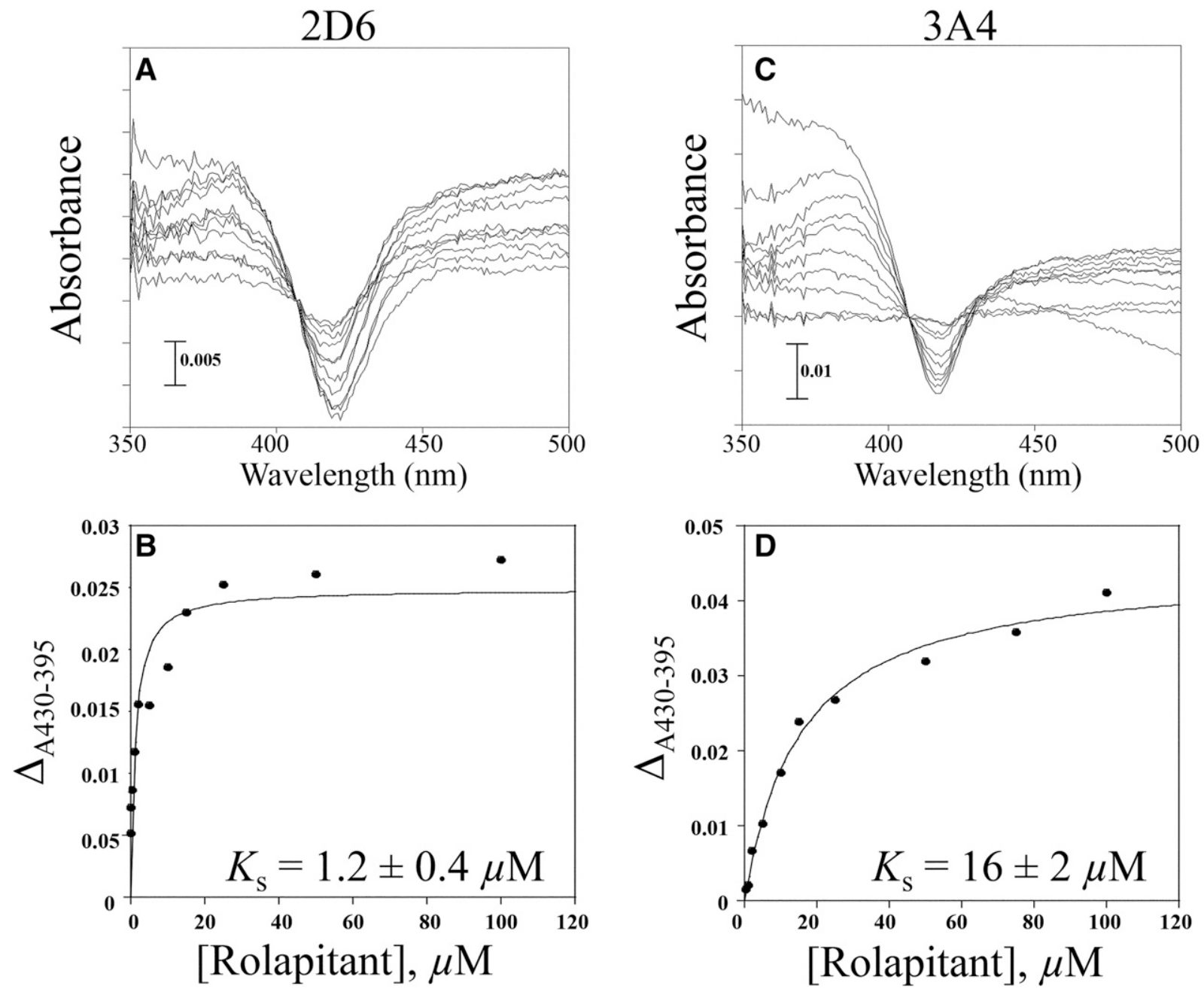

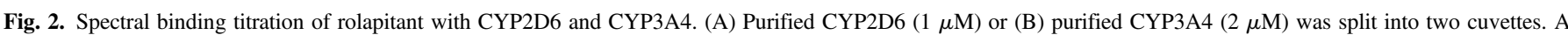

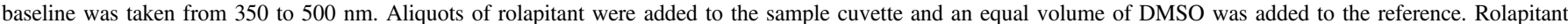

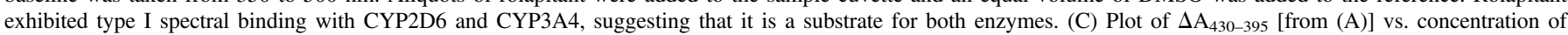

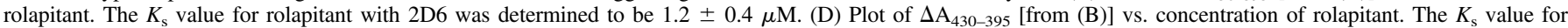
rolapitant with $3 \mathrm{~A} 4$ was determined to be $16 \pm 2 \mu \mathrm{M}$. 


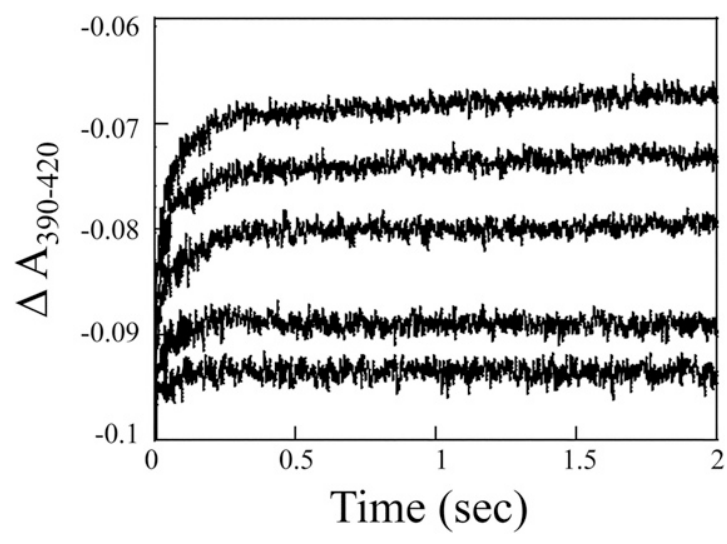

Fig. 3. Determination of $K_{\mathrm{d}}$ by stopped-flow spectroscopy. Composite absorbance changes from absorbance maxima and minima $\left(\Delta \mathrm{A}_{390-420}\right)$ over time (4 seconds) were created for each rolapitant concentration $(1,5,10,25$, and $50 \mu \mathrm{M})$. Only the first 2 seconds are shown. At least three replicates of each ligand concentration were averaged and plotted over time. Values for $k_{\text {on }}$ and $k_{\text {off }}\left(1.12 \times 10^{6} \mathrm{M}^{-1} \mathrm{~s}^{-1}\right.$ and 7.04 second ${ }^{-1}$, respectively), were determined using KinTek Global Kinetic Explorer software. The $K_{\mathrm{d}}$ value was determined to be $6.2 \mu \mathrm{M}$ by the ratio of $k_{\text {off }} / k_{\text {on }}$.

track redundant or superfluous motion, but did track overall rolapitant movement. The reference point near the heme was picked to identify alternative low-energy binding poses with higher resolution. Additionally, monitoring the accumulation of the energy deposition that is required to get the ligand to move from a particular location can be used to estimate the free energy at that location (Supplemental Fig. 1, F and G).

Free energy binding landscapes produced from the adaptive biasing simulations were visualized using the R 3.2.3 statistical framework (R Core Team, 2017) with the lattice library version 0.20.23 (Sarkar, 2008). Rolapitant egress trajectories and molecular images were produced using VMD version 1.9.3 (University of Illinois, Urbana-Champaign, IL).

\section{Results}

In spectral binding titrations, rolapitant displayed type I spectral binding with respect to binding both CYP2D6 and CYP3A4, as observed by an increase in absorbance at $390 \mathrm{~nm}$ and a decrease at $420 \mathrm{~nm}$ (Fig. 2). Spectral binding affinity $\left(K_{\mathrm{s}}\right)$ was calculated to be $1.2 \pm$ $0.4 \mu \mathrm{M}$ for CYP2D6 and $16 \pm 2 \mu \mathrm{M}$ for CYP3A4, indicating much tighter binding to CYP2D6 (Fig. 2). Based on this observation, we hypothesized that perhaps inhibition of CYP2D6 in vivo might be due to a slow off rate for rolapitant from CYP2D6. Stopped-flow measurement of $k_{\text {on }}$ and $k_{\text {off }}$ rates for rolapitant from CYP2D6 yielded a $k_{\text {on }}$ value of $1.12 \times 10^{6} \mathrm{M}^{-1} \mathrm{~s}^{-1}$ and $k_{\text {off }}$ value of 7.04 second $^{-1}$ using a global exponential fit (Fig. 3). A $k_{\text {off }}$ value of 7.04 second $^{-1}$ indicates a $t_{\mathrm{R}}$ value of 0.142 seconds for rolapitant with CYP2D6. The $K_{\mathrm{d}}$ value for rolapitant with CYP2D6 calculated from $k_{\text {off }} / k_{\text {on }}$ was $6.2 \mu \mathrm{M}$, which was similar to the observed $K_{\mathrm{s}}$.

Time- and concentration-dependent inactivation assays showed that rolapitant did not behave as a mechanism-based inactivator of CYP2D6 (Supplemental Fig. 2). Also, no metabolites of rolapitant reactions with CYP2D6 were observed (data not shown). To test possible inactivation of CYP2D6 by metabolites of CYP3A4 or other drug-metabolizing enzymes, coincubation assays containing either mixed CYP3A4 with CYP2D6 supersomes or human liver microsomes mixed with CYP2D6 supersomes were completed (data not shown). Over time, no inactivation of CYP2D6 was observed under these conditions, suggesting that rolapitant is not metabolized to another form that could serve to inactivate CYP2D6.

A further possibility was that rolapitant could be a tight-binding reversible inhibitor of CYP2D6. The drug quinidine is a classic example of a tight-binding inhibitor of CYP2D6 that strongly inhibits the activity

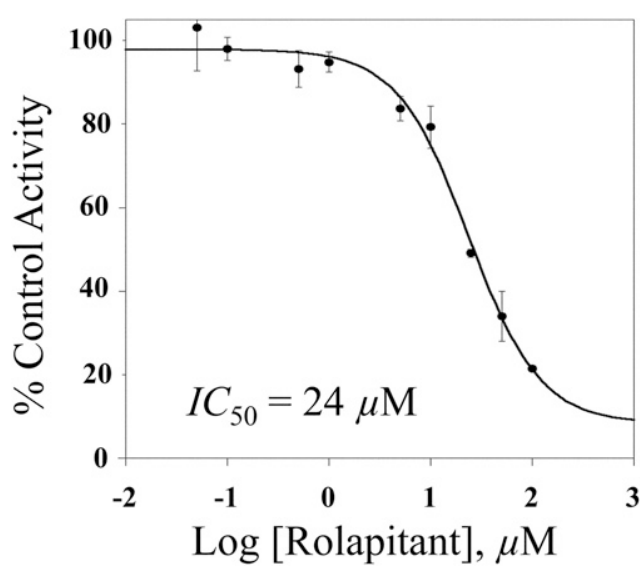

Fig. 4. Determination of $\mathrm{IC}_{50}$ for rolapitant inhibition of CYP2D6. CYP2D6 supersomes $(2 \mathrm{pmol})$ were incubated with dextromethorphan $(10 \mu \mathrm{M})$ and varying concentrations of rolapitant $(0.05-100 \mu \mathrm{M})$. Log concentration is plotted against percentage of activity of dextrorphan product formation relative to a $0 \mu \mathrm{M}$ inhibitor control. The $\mathrm{IC}_{50}$ value was $24 \mu \mathrm{M}$ for rolapitant. Each point represents the mean \pm S.D. of an experiment completed in triplicate.

of CYP2D6, but does not have a long in vivo half-life (elimination of 68 hours) (Zhou, 2009). In $\mathrm{IC}_{50}$ assays, quinidine showed a biologically relevant $\mathrm{IC}_{50}$ value of $0.06 \mu \mathrm{M}$ (e.g., $60 \mathrm{nM}$ ), similar to values previously reported (Hutzler et al., 2003) (Supplemental Fig. 3). In comparison, the $\mathrm{IC}_{50}$ for rolapitant was three orders of magnitude higher at $24 \mu \mathrm{M}$ (Fig. 4).

Given that reversible inhibition was observed, Lineweaver-Burk and Dixon analyses were completed to determine the type and extent of inhibition. From Lineweaver-Burk analysis with two different substrates (dextromethorphan and bufuralol), mixed inhibition was observed (Fig. 5). The $K_{\mathrm{m}}$ and $V_{\max }$ values for either dextromethorphan or bufuralol metabolism were altered with increasing concentrations of rolapitant in the reactions; the $K_{\mathrm{m}}$ values increased roughly 2- to 3 -fold, while the $V_{\max }$ value was approximately halved. Plotting of the kinetic data in Michaelis-Menten-style plots did not reveal any reporter substrate inhibition of CYP2D6 over the range of substrate concentrations used in our analysis (data not shown). The Dixon analysis yielded $K_{\mathrm{i}}$ values of 20 and $34 \mu \mathrm{M}$ with bufuralol and dextromethorphan, respectively, as reporter substrates (Fig. 6).

Using the $K_{\mathrm{i}}$ values from the Dixon analysis, static drug-drug interactions were modeled using the 2017 FDA DDI guidance for the industry predicted ratio calculation of $\mathrm{R} 1=1+\left([\mathrm{I}]_{\max , \mathrm{u}} / K_{\mathrm{i}}\right)($ Food and Drug Administration, 2017), where R1 is the predicted ratio of the victim drug's area under the curve in the presence and absence of rolapitant, $[\mathrm{I}]_{\max , \mathrm{u}}$ is the concentration of rolapitant unbound, and $K_{\mathrm{i}}$ is the inhibition constant. In vivo, after a single $180 \mathrm{mg}$ dose, the $C_{\max }$ value of rolapitant has been reported to be $\sim 2.5 \mu \mathrm{M}$ and rolapitant is $\sim 99 \%$ bound in the plasma (Wang et al., 2017). For DDI calculations, only unbound drug concentration as used. From this, the R1 value for rolapitant was $\sim 1.0$. This value, according to FDA guidance, does not predict DDI with CYP2D6. We note that using DDI calculation standards prior to 2017 , e.g., at the time rolapitant was originally evaluated by the FDA, DDI modeling calculations used total drug concentration at $C_{\max }(\sim 2.5 \mu \mathrm{M}$ for rolapitant) and slight DDI were predicted. For comparison with rolapitant, we did the same R1 calculations with the $\mathrm{IC}_{50}$ data we generated with quinidine and literature values for quindine $C_{\max }$. In the case of quinidine, R1 values over 7 were calculated, consistent with strong inhibition as observed in vivo.

To better understand the interactions of rolapitant with CYP2D6, ten 1-microsecond molecular dynamics simulations were performed using 
A

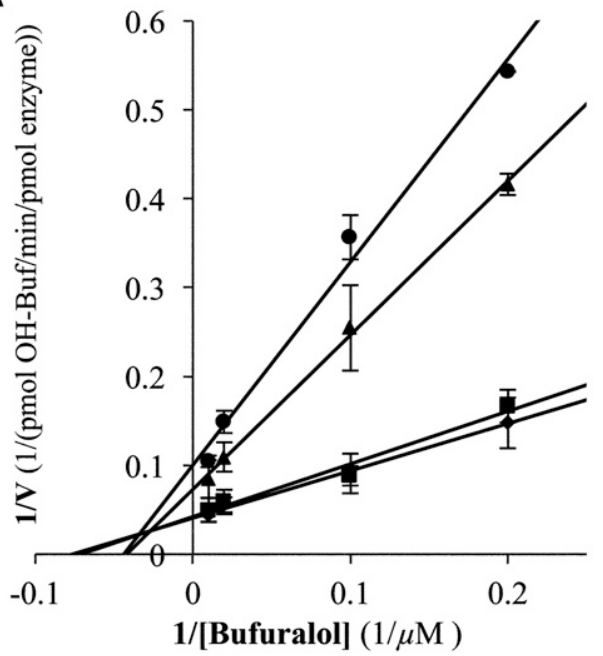

B

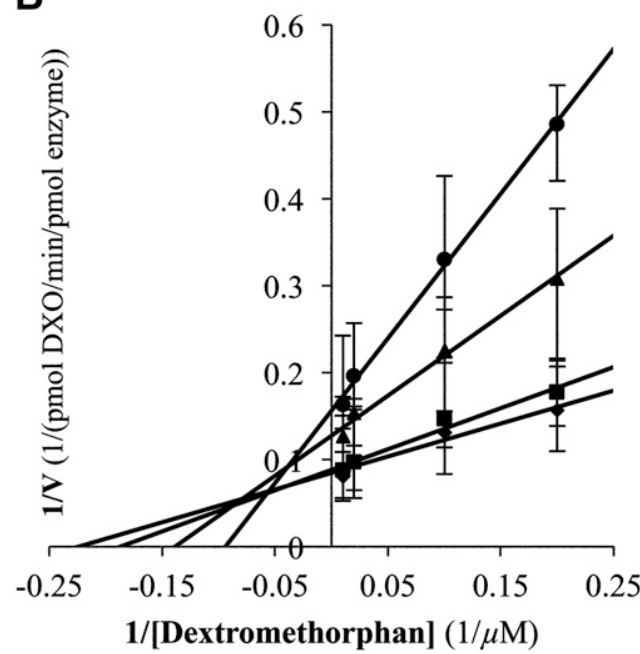

Fig. 5. Lineweaver-Burk analysis of the inhibitory effect of rolapitant on CYP2D6. Metabolism of dextromethorphan (A) or bufuralol (B) at four different concentrations $(5,10,50$, and $100 \mu \mathrm{M})$ was examined in the presence of $0(\diamond), 10(\boldsymbol{\square})$, $50(\mathbf{\Delta})$, and $100 \mu \mathrm{M}(\mathbf{)})$ rolapitant. Each data point represents an average of an experiment completed in quadruplicate. In reactions with dextromethorphan and bufuralol, the $K_{\mathrm{m}}$ value increased with increasing concentrations of rolapitant and the $V_{\max }$ value was also affected, indicating mixed inhibition.

approximately 100 days of GPU compute time. For rolapitant and CYP2D6, the $t_{\mathrm{R}}$ value was 0.142 seconds, or $1.42 \times 10^{5}$ microseconds based on the $k_{\text {off }}$ value measured in the previous stopped-flow experiments. To compensate for the disparity between the residence time $\left(1.42 \times 10^{5}\right.$ microseconds $)$ and our simulation times ( 1 microsecond), we examined ligand binding and egress using molecular dynamics simulations modified to include an adaptive biasing potential as described in Materials and Methods.

Rolapitant remained fully enclosed in the active site in six simulations and moved outside the protein in four simulations (Supplemental Fig. 4; Supplemental Material) via channels defined by the nomenclature of Cojocaru et al. (2007) (Fig. 7). In our 1-microsecond simulations, rolapitant never reentered the enzyme after egressing. Most commonly, protonated rolapitant bound in a fully extended pose in a pocket that was within $5 \AA$ of mostly hydrophobic residues. There were some indications of $\pi-\pi$ interactions between rolapitant and Phe247 and Phe112, but the conformations for interactions were not consistent or strong (e.g., no $\pi-\pi$ stacking) (Supplemental Figs. 4 and 5 and data not shown).
A

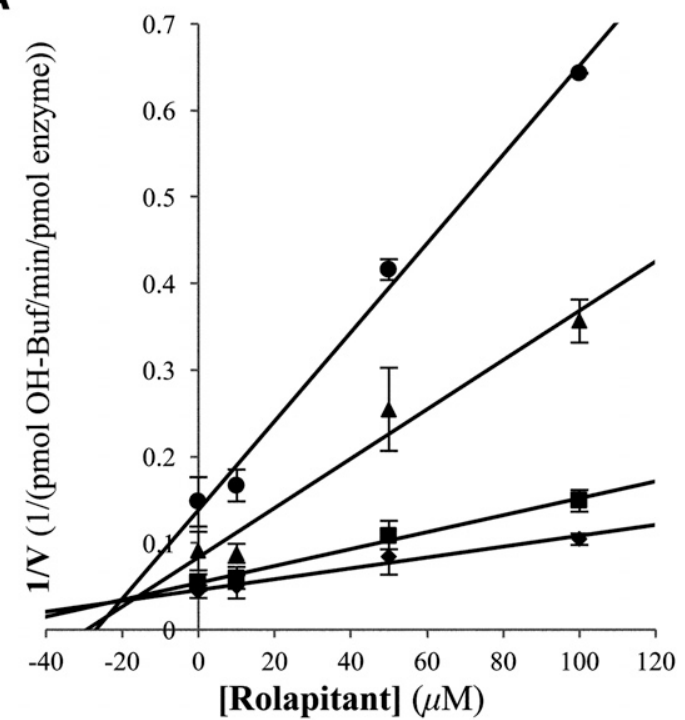

B

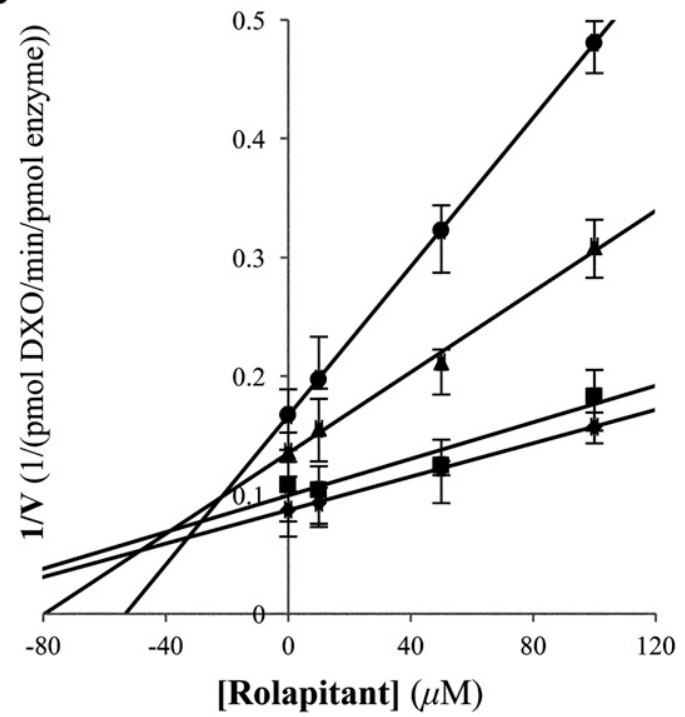

Fig. 6. Dixon analysis of rolapitant inhibition of CYP2D6. The concentration of rolapitant was plotted vs. the inverse velocity for each line of dextromethorphan (A) or bufuralol (B) at concentrations of $5(\boldsymbol{O}), 10(\mathbf{\Delta}), 50(\boldsymbol{\square})$, and $100 \mu \mathrm{M}(\diamond)$ to yield $K_{\mathrm{i}}$ values of 34 and $20 \mu \mathrm{M}$ in assays with dextromethorphan and bufuralol, respectively. Each data point represents an average of an experiment completed in quadruplicate.

Movement of Phe483 allowed access of rolapitant to the solvent channel (data not shown). Overall, both the low-energy binding poses and egress through the $2 \mathrm{c}$ channel were consistent with rolapitant behaving as a reversible inhibitor. We also noted that in all simulations, rolapitant was completely above the active site and outside the range of metabolism.

\section{Discussion}

In vivo, rolapitant inhibits the activity of CYP2D6 for at least 7 days following a single dose. Rolapitant is one of the largest ligands known to bind CYP2D6 and its mechanism of multiday in vivo inhibition is unknown. In this study, we examined several possible mechanisms for inhibition, including possible inactivation.

The spectral binding constant for rolapitant with CYP2D6 $(1.2 \pm 0.4$ $\mu \mathrm{M})$ was in a similar range as seen with other CYP2D6 ligands, including several mechanism-based inactivators previously reported 


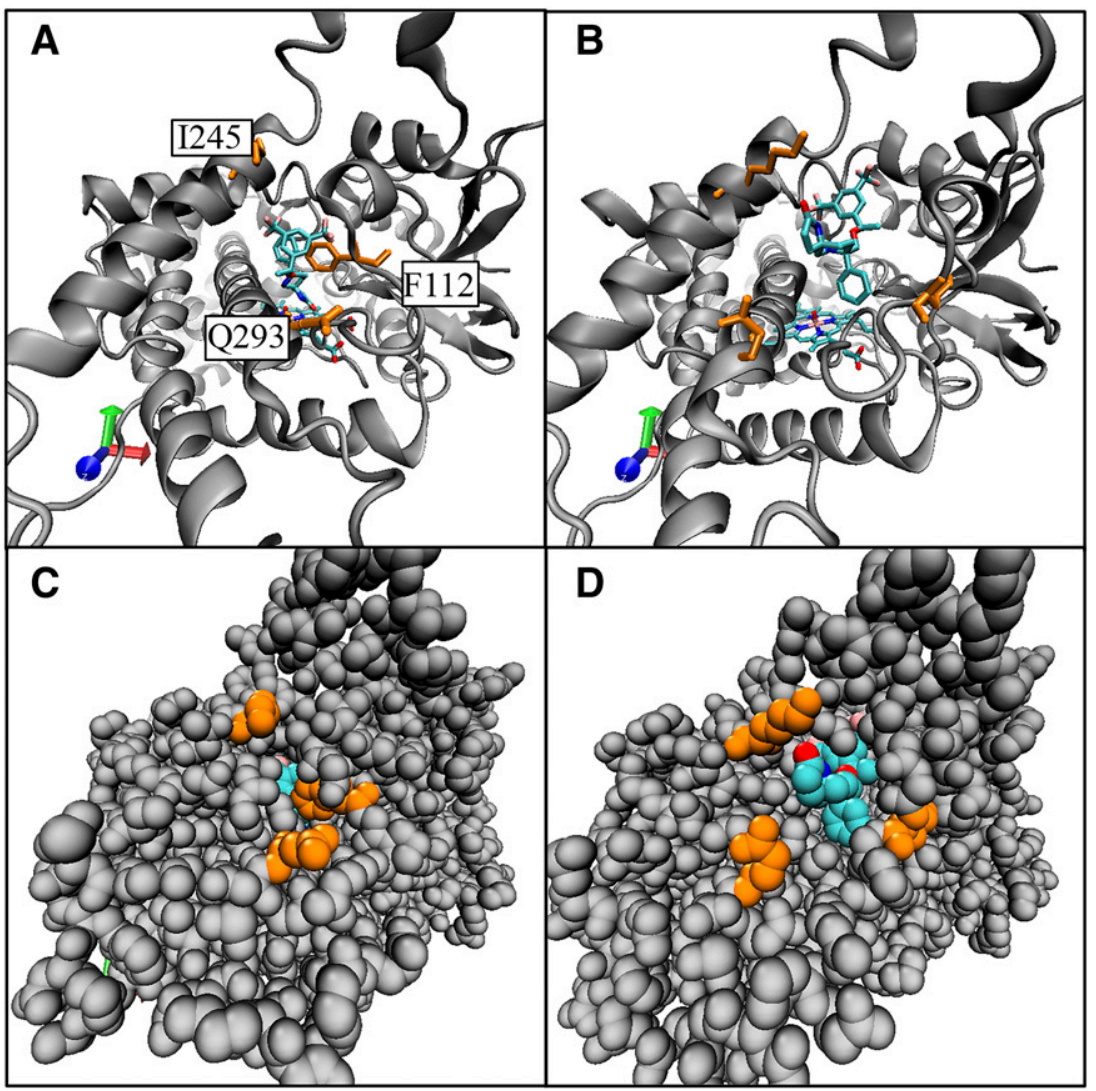

Fig. 7. Comparison of the active site starting position to the egress position in channel $2 \mathrm{c}$ for rolapitant egress. Frames from molecular dynamics simulation 2 comparing the starting position of rolapitant at the active site of CYP2D6 to the egress position at the opening of channel $2 \mathrm{c}$ are shown. Rolapitant is shown in turquoise. Select amino acids along channel 2c are shown in orange: Phe112 on the helix B'/B-C loop, Glu293 on the $N$-terminal end of helix I, and Lys 245 on helix G. (A) Initial position for rolapitant in the active site of CYP2D6 in a ribbon model. (B) Frame where rolapitant egresses from CYP2D6 via channel 2c. (C). Space-filling model of structure in (A); rolapitant is only slightly visible in the active site. (D). Space-filling model of structure in (B) with rolapitant emerged from channel 2c.

( $\sim 0.4-30 \mu \mathrm{M})$ (Nagy et al., 2011; Livezey et al., 2014). The low value for the spectral binding constant could lead to observed inhibition if the concentration of rolapitant was high enough in vivo. A single $180 \mathrm{mg}$ dose of rolapitant produces a $C_{\max }$ value of $\sim 2.4 \mu \mathrm{M}$ in vivo (Wang et al., 2017). However, the $\mathrm{IC}_{50}$ and $K_{\mathrm{i}}$ values for rolapitant $(24 \mu \mathrm{M}$ for $\mathrm{IC}_{50}$ and 20 and $34 \mu \mathrm{M}$ for $K_{\mathrm{i}}$, depending on substrate reporter) are greater than $C_{\max }$ in vivo concentrations of rolapitant. The $K_{\mathrm{i}}$ values for rolapitant are also similar to those reported for inhibition of CYP2D6 by amiodarone (a ligand inhibitor larger than rolapitant) and metoprolol (a smaller ligand inhibitor): 26.8 and $11.8 \mu \mathrm{M}$ for amiodarone and 17.0 and $11.3 \mu \mathrm{M}$ for metoprolol with bufuralol and dextromethorphan, respectively, as reporter substrates (VandenBrink et al., 2012)]. There was no observed metabolism of rolapitant by CYP2D6 and no time- or concentration-dependent inactivation (Supplemental Fig. 2; data not shown). CYP2D6 was also not inhibited in assays that included CYP3A4 and rolapitant (data not shown). Although the concentrations of M19 in the assays would be low, the lack of inhibition was consistent with a recent in vivo clinical study with rolapitant that suggested the M19 formed by CYP3A4 would not be a strong inhibitor of CYP2D6 (Wang et al., 2019). The $k_{\text {on }}$ and $k_{\text {off }}$ values calculated from the stoppedflow studies $\left(1.12 \times 10^{6} \mathrm{M}^{-1} \mathrm{~s}^{-1}\right.$ and 7.04 second $^{-1}$, respectively) were similar to those reported by Yun et al. (2005) for 7-OH coumarin binding to CYP2A6 $\left(k_{\text {on }}\right.$ of $2.0 \times 10^{6} \mathrm{M}^{-1} \mathrm{~s}^{-1}, k_{\text {off }}$ of 6.8 second $^{-1}$, and $k_{\text {off }} / k_{\text {on }}=3.4 \mu \mathrm{M} ; t_{\mathrm{R}}=0.147$ seconds), and overall did not suggest unusual individual rate constants as an explanation for inhibition.

To understand possible binding modes that might lead to inhibition, adaptive biasing molecular dynamics simulations were completed. In our simulations, both Asp301 and Glu216 acted as binding residues for rolapitant, but the ligand was outside the metabolism range of the heme consistent with a lack of metabolism in in vitro studies. We also observed that Phe 483 could play a role in directionality of ligand egress, particularly relevant to the solvent channel, as suggested in crystal structures by Wang et al. (2015).

The hydrophobic binding area in our simulations was the same as those described in the crystal structures of CYP2D6 including both Rowland et al. (2006) and Wang et al. (2012, 2015) and in docking studies with CYP2D6 inhibitors and substrates by VandenBrink et al. (2012), who further suggest that interactions in this binding pocket may influence inhibition. Similarly, in polymorphic forms of CYP2C9, Maekawa et al. (2017) found three different ligand binding sites-a peripheral site, an active site, and an access channel site - and suggested that the access channel site might be a site for regulation of allosteric inhibition. Siu et al. (2018) recently described an allosteric binding antechamber that was consistent with stabilization of CYP2D6 inhibition by celecoxib. Our findings, along with other previous findings, support the hypothesis that similar binding sites may be present in CYP2D6 as well, and that binding in these sites may serve an inhibitory function.

Overall, the molecular dynamics studies support plasticity in P450s and support a model that ligands, particularly large ones, egress from CYP2D6 via multiple small-scale fluctuations rather than large swings or rotations. This is consistent with the suggestion by Rowland et al. (2006) in the first crystal structure of CYP2D6 that such fluctuations would be normal and necessary for ligand movement within protein channels. In addition, since rolapitant was not a mechanism-based inactivator or tight-binding inhibitor of CYP2D6, the observed in vivo inhibition of CYP2D6 by rolapitant may be simply due to the long 7-day in vivo half-life.

\section{Acknowledgments}

We thank Parker de Waal and Dr. Brad Dickson for helpful discussions involving the implementation and interpretation of the molecular dynamics 
experiments. We thank Dr. Fred Guengerich for assistance with the stopped-flow experiments and global fitting with KinTek software. We thank Emily Olson Dumas and Daniel Bow for helpful discussions of DDI modeling.

\section{Authorship Contributions}

Participated in research design: Glass, Leddy, Orwin, K. A. Furge, L. L. Furge.

Conducted experiments: Glass, Leddy, Orwin, Miller, K. A. Furge, L. L. Furge.

Performed data analysis: Glass, Leddy, Orwin, K. A. Furge, L. L. Furge.

Wrote or contributed to the writing of the manuscript: Glass, Leddy, K. A. Furge, L. L. Furge.

\section{References}

Chico LK, Behanna HA, Hu W, Zhong G, Roy SM, and Watterson DM (2009) Molecular properties and CYP2D6 substrates: central nervous system therapeutics case study and pattern analysis of a substrate database. Drug Metab Dispos 37:2204-2211.

Cojocaru V, Winn PJ, and Wade RC (2007) The ins and outs of cytochrome P450s. Biochim Biophys Acta 1770:390-401.

de Waal PW, Sunden KF, and Furge LL (2014) Molecular dynamics of CYP2D6 polymorphisms in the absence and presence of a mechanism-based inactivator reveals changes in local flexibility and dominant substrate access channels. PLoS One 9:e108607.

Dickson BM, de Waal PW, Ramjan ZH, Xu HE, and Rothbart SB (2016) A fast, open source implementation of adaptive biasing potentials uncovers a ligand design strategy for the chromatin regulator BRD4. J Chem Phys 145:154113.

Food and Drug Administration (Press release, Sept. 1, 2015) FDA approves new drug treatment for nausea and vomiting from chemotherapy, Food and Drug Administration, Silver Spring, MD.

Food and Drug Administration (2017) In Vitro Metabolism and Transporter-Medicated Drug-Drug Interaction Studies: Guidance for Industry, Food and Drug Administration, Silver Spring, MD.

Gillam EMJ, Baba T, Kim BR, Ohmori S, and Guengerich FP (1993) Expression of modified human cytochrome P450 3A4 in Escherichia coli and purification and reconstitution of the enzyme. Arch Biochem Biophys 305:123-131.

Gillam EMJ, Guo Z, Ueng YF, Yamazaki H, Cock I, Reilly PEB, Hooper WD, and Guengerich FP (1995) Expression of cytochrome P450 3A5 in Escherichia coli: effects of 5' modification, purification, spectral characterization, reconstitution conditions, and catalytic activities. Arch Biochem Biophys 317:374-384

Glass SM, Martell CM, Oswalt AK, Osorio-Vasquez V, Cho C, Hicks MJ, Mills JM, Fujiwara R, Glista MJ, Kamath SS, et al. (2018) CYP2D6 allelic variants *34, *17-2, *17-3, and *53 and a Thr309Ala mutant display altered kinetics and NADPH coupling in metabolism of bufuralol and dextromethorphan and altered susceptibility to inactivation by SCH 66712. Drug Metab Dispos 46:1106-1117.

Guengerich FP (2015) Human cytochrome P450 enzymes, in Cytochrome P450: Structure, Mechanism, and Biochemistry (Ortiz de Montellano PR ed) pp 523-786, Springer, New York.

Hanna IH, Kim MS, and Guengerich FP (2001) Heterologous expression of cytochrome P450 2D6 mutants, electron transfer, and catalysis of bufuralol hydroxylation: the role of aspartate 301 in structural integrity. Arch Biochem Biophys 393:255-261.

Huey R, Morris GM, Olson AJ, and Goodsell DS (2007) A semiempirical free energy force field with charge-based desolvation. J Comput Chem 28:1145-1152.

Hutzler JM, Walker GS, and Wienkers LC (2003) Inhibition of cytochrome P450 2D6: structureactivity studies using a series of quinidine and quinine analogues. Chem Res Toxicol 16:450-459.

Livezey MR, Briggs ED, Bolles AK, Nagy LD, Fujiwara R, and Furge LL (2014) Metoclopramide is metabolized by CYP2D6 and is a reversible inhibitor, but not inactivator, of CYP2D6. Xenobiotica 44:309-319.
Maekawa K, Adachi M, Matsuzawa Y, Zhang Q, Kuroki R, Saito Y, and Shah MB (2017) Structural basis of single-nucleotide polymorphisms in cytochrome P450 2C9. Biochemistry 56: 5476-5480.

Morris GM, Goodsell DS, Halliday RS, Huey R, Hart WE, Belew RK, and Olson AJ (1998) Automated docking using a Lamarckian genetic algorithm and an empirical binding free energy function. J Comput Chem 19:1639-1662.

Nagy LD, Mocny CS, Diffenderfer LE, Hsi DJ, Butler BF, Arthur EJ, Fletke KJ, Palamanda JR, Nomeir AA, and Furge LL (2011) Substituted imidazole of 5-fluoro-2-[4-[(2-phenyl-1 Himidazol-5-yl)methyl]-1-piperazinyl]pyrimidine inactivates cytochrome P450 2D6 by protein adduction. Drug Metab Dispos 39:974-983.

Olver I (2015) Role of rolapitant in chemotherapy-induced emesis. Lancet Oncol 16:1006-1007.

Poma A, Christensen J, Pertikis H, Arora S, and Hedley M (2013) Rolapitant and its major metabolite do not affect the pharmacokinetics of midazolam, a sensitive cytochrome P450 3A4 substrate (Abstract 441). Support Care Cancer 21:S154.

R Core Team (2017) R: A Language and Environment for Statistical Computing, R Foundation for Statistical Computing, Vienna, Austria.

Rowland P, Blaney FE, Smyth MG, Jones JJ, Leydon VR, Oxbrow AK, Lewis CJ, Tennant MG, Modi S, Eggleston DS, et al. (2006) Crystal structure of human cytochrome P450 2D6. J Bio Chem 281:7614-7622.

Sarkar D (2008) Lattice: Multivariate Data Visualization with R, Springer, New York.

Shahrokh K, Orendt A, Yost GS, and Cheatham TE III (2012) Quantum mechanically derived AMBER-compatible heme parameters for various states of the cytochrome P450 catalytic cycle. $J$ Comput Chem 33:119-133

Siu YA, Hao MH, Dixit V, and Lai WG (2018) Celecoxib is a substrate of CYP2D6: impact on celecoxib metabolism in individuals with CYP2C $9 * 3$ variants. Drug Metab Pharmacokinet 33 219-227.

Trott $\mathrm{O}$ and Olson AJ (2010) AutoDock Vina: improving the speed and accuracy of docking with a new scoring function, efficient optimization, and multithreading. $J$ Comput Chem 31:455-461.

VandenBrink BM, Foti RS, Rock DA, Wienkers LC, and Wahlstrom JL (2012) Prediction of CYP2D6 drug interactions from in vitro data: evidence for substrate-dependent inhibition. Drug Metab Dispos 40:47-53.

Vanquelef E, Simon S, Marquant G, Garcia E, Klimerak G, Delepine JC, Cieplak P, and Dupradeau FY (2011) R.E.D. Server: a web service for deriving RESP and ESP charges and building force field libraries for new molecules and molecular fragments. Nucleic Acids Res 39:W511-W517. Varubi (2015) Package insert. TESARO, Waltham, MA

Wang A, Savas U, Hsu MH, Stout CD, and Johnson EF (2012) Crystal structure of human cytochrome P450 2D6 with prinomastat bound. J Biol Chem 287:10834-10843.

Wang A, Stout CD, Zhang Q, and Johnson EF (2015) Contributions of ionic interactions and protein dynamics to cytochrome P450 2D6 (CYP2D6) substrate and inhibitor binding. J Biol Chem 290:5092-5104.

Wang J, Zhang ZY, Lu S, Powers D, Kansra V, and Wang X (2019) Effects of rolapitant administered orally on the pharmacokinetics of dextromethorphan (CYP2D6), tolbutamide (CYP2C9), omeprazole (CYP2C19), efavirenz (CYP2B6), and repaglinide (CYP2C8) in healthy subjects. Support Care Cancer 27:819-827.

Wang X, Zhang ZY, Powers D, Wang J, Lu S, and Kansra V (2017) Rolapitant absolute bioavailability and PET imaging studies in healthy adult volunteers. Clin Pharmacol Ther 102: 332-339.

Yun CH, Kim KH, Calcutt MW, and Guengerich FP (2005) Kinetic analysis of oxidation of coumarins by human cytochrome P450 2A6. J Biol Chem 280:12279-12291.

Zhou SF (2009) Polymorphism of human cytochrome P450 2D6 and its clinical significance: part I. Clin Pharmacokinet 48:689-723.

Address correspondence to: Laura Lowe Furge, Department of Chemistry, Kalamazoo College, 1200 Academy Street, Kalamazoo, MI 49006. E-mail: Laura.Furge@kzoo.edu 